\title{
The contribution of patient interviews to the identification of drug-related problems in home medication review
}

\author{
H. F. Kwint ${ }^{*} \uparrow$ PharmD, A. Faber $\uparrow$ PhD, J. Gusseklooł PhD, MD and M. L. Bouvy ${ }^{*} \uparrow \mathrm{PhD}$ \\ ${ }^{*}$ Division of Pharmacoepidemiology \& Clinical Pharmacology, Utrecht Institute for Pharmaceutical Sciences (UIPS), Utrecht, †SIR Institute for Pharmacy \\ Practice and Policy, Leiden, and $\$$ Department of Public Health and Primary Care, Leiden University Medical Center, Leiden, The Netherlands
}

Received 25 January 2012, Accepted 26 June 2012

Keywords: drug-related problems, home visits, medication review, patient interviews

\section{SUMMARY}

What is known and Objective: To determine to what extent patient interviews contribute to the identification of drugrelated problems (DRPs) in home medication reviews, in terms of number, type and clinical relevance.

Methods: We performed a cross-sectional study within the intervention arm of a randomized controlled trial. Patients were recruited from 10 Dutch community pharmacies. Patients were eligible if they were home-dwelling, aged 65 years and over and used five or more different drugs, including at least one cardiovascular or antidiabetic drug. The community pharmacist interviewed the patient at home about the medicines and identified potential DRPs in combination with medication and clinical records. This medication review was assessed and modified by an independent pharmacist reviewers' panel. Outcomes were the number and type of DRPs and recommendations and percentage of clinical relevant DRPs. Clinical relevance of DRPs was assessed by DRPs assigned a high priority, DRPs followed by recommendations for drug change and DRPs followed by implemented recommendations for drug change.

Results: A total of 1565 potential DRPs and recommendations (10 per patient).were identified for 155 patients (median age, 76 years; $54 \%$ women). Fifty-eight per cent of all recommendations involved a drug change; $27 \%$ of all DRPs were identified during patient interviews and $74 \%$ from medication and clinical records. Compared to DRPs identified from patient medication and clinical records, DRPs identified during patient interviews were more frequently assigned a high priority $(\mathrm{OR}=1 \cdot 8[1 \cdot 4-$ $2 \cdot 2]$ ), were more frequently associated with recommendations for drug change $(\mathrm{OR}=2.4$ [1.9-3.1]) and were implemented recommendations for drug change (OR $=2 \cdot 8$ [2:1-3.7]).

What is new and Conclusion: This study shows that more than a quarter of all DRPs were identified during patient interviews. DRPs identified during patient interviews were more frequently assigned a higher clinical relevance.

\section{WHAT IS KNOWN AND OBJECTIVE}

Studies have identified potential drug-related problems (DRPs) during different types of medication review. ${ }^{1-8}$ Medication

Correspondence: H.F. Kwint, Division of Pharmacoepidemiology \& Clinical Pharmacology, Utrecht Institute for Pharmaceutical Sciences (UIPS), Utrecht, The Netherlands. Tel.: +0031715766311; fax +0031715766251; e-mail: h.f.kwint@uu.nl reviews can be solely based on patient medication and clinical records, ${ }^{1,2}$ but can also be combined with patient interviews. ${ }^{3-8}$ This more extensive review is known as a clinical medication review. ${ }^{9}$

Patient interviews have been performed in several settings such as hospitals, ${ }^{3}$ pharmacies, ${ }^{8}$ GPs' $^{\prime}$ offices ${ }^{4,7}$ or at the patient's home. ${ }^{5,6}$ In Australia, a patient interview at home by an accredited pharmacist is the predominant method of clinical medication review. ${ }^{5,10-12}$ In Europe, the patient is often invited to the community pharmacy for an interview, as with the Medicine Use Review (MUR) in England. ${ }^{13}$ Different European studies in primary care included patient interviews at home, ${ }^{6,14-16}$ but these were not always conducted by a clinically well-trained pharmacist and in close cooperation with a GP, like in Australia. ${ }^{14-16}$

Although DRPs identified during patient interviews have been shown to be clinically relevant, it is unclear to what extent additional DRPs are identified when complete clinical and medication records are available. Moreover, limited knowledge is available on the clinical relevance of DRPs identified through patient interviews compared to DRPs identified from clinical and medication records. ${ }^{3}$

This study examines the contribution of a patient interview to the identification of DRPs in home medication review with the availability of complete dispensing and clinical records. Moreover, we aimed to compare the clinical relevance of DRPs identified during patient interviews to DRPs identified by the combination of patient medication and clinical records.

\section{METHODS}

\section{Study design}

This was a cross-sectional study within the intervention arm of a randomized controlled trial (RCT) in a primary care setting. This RCT aimed to assess whether home medication reviews could reduce the number of DRPs and increase the proportion of patients with adequate control of blood pressure, cholesterol and $\mathrm{HbA} 1 \mathrm{C}$ values. Patients were enrolled between February 2008 and August 2010.

\section{Patients}

Patients were recruited from 10 Dutch community pharmacies. Patients were eligible if they were home-dwelling, aged 65 years and over and used at least five oral prescription 
drugs, including at least one cardiovascular or one antidiabetic drug. Consenting patients were visited and interviewed face-to-face by a pharmacist at home. Patients were excluded if the majority of drugs were prescribed by specialists.

Formal ethical approval was obtained from the medical ethical review board of the Utrecht University Medical Centre. Patients gave written informed consent. To protect the patient's privacy, all medical data were anonymized by the community pharmacist using a randomly assigned unique number for each patient.

\section{Intervention}

For all patients, complete patient medication records from the community pharmacy including drug dispensing records, information on comorbidity, drug intolerances and other relevant patient notes were available. Because the majority of patients in the Netherlands are registered at only one community pharmacy, independently of prescriber, patient medication records are virtually complete with regard to prescription drugs. ${ }^{17}$ The community pharmacists collected data from the clinical records of the patient with the help from the GP practice, including medical history and laboratory data. As part of the study protocol, patients were offered additional laboratory measurements of $\mathrm{HbA1C}$, cholesterol, sodium, potassium and creatinine and blood pressure measurement. The patient's community pharmacist interviewed the patient at home aiming to identify possible DRPs.

During the home visit, the community pharmacist evaluated all medications that patients kept at home, including discontinued prescription drugs, over-the-counter drugs and complementary and alternative medicines (patients indicated whether each medication was currently taken). Community pharmacists had limited experience with medication review. Therefore, they received a 2-day training course in medication review as a part of this study. Within this course, pharmacists were taught how to perform a structured medication review and how to communicate with patients about adherence and understanding of the drug therapy regimen and about patient's experiences and concerns regarding drug therapy (in particular, possible adverse effects). GPs did not receive additional training in medication review.

A pharmaceutical care plan was proposed by the community pharmacist using both the patient medication and clinical records (including additional laboratory data and blood pressure collected as part of the study protocol) and the data from the patient interview. These pharmaceutical care plans were evaluated, if necessary adjusted, and completed by two independent pharmacists from a pool of three pharmacist reviewers (A.F; J.K.D; and H.K.). Pharmacist reviewers had several years of experience with medication review, as well as in-depth knowledge of national clinical guidelines.

Reviewers used both implicit and explicit criteria to identify potential DRPs. Explicit criteria consisted of a list of clinical rules based on Dutch treatment and prescription guidelines. Examples of clinical rules were 'Lack of appropriate treatment for secondary prevention for CHD (antiplatelet, lipid-lowering, $\beta$-blocker, ACE-inhibitor)', 'Lack of appropriate treatment for patients with diabetes and LDL $>2.5 \mathrm{mmol}$ (lipid lowering)' or 'No available monitoring data for blood pressure, lipids, glucose, BMI or data $>1$ year old in patients for whom these measurements are indicated in treatment guidelines'. ${ }^{18}$

Implicit criteria for identifying DRPs were based on a structural assessment as proposed by Cipolle et al. in the rational order of indication, effectiveness, safety and compliance. ${ }^{19,20}$ The two reviewers reached consensus in a case conference. If no consensus was met, the third reviewer was consulted until consensus was reached. For example, when the two reviewers could not agree (e.g. on the necessity of gastric protection with a proton pump inhibitor in a geriatric patient using aspirin or on the need to change the dosing time for simvastatin from the afternoon to the evening).

Drug-related problems were prioritized by the pharmacist reviewers as high, medium or low from the patient's perspective, with the highest priority on those that cause the most con$\operatorname{cern}^{20}$ and need action. For example, high priority was assigned to recommendations that could directly relief patient complaints or to recommendations following on measurement of a deviating laboratory value or blood pressure.

The pharmaceutical care plans were sent to the community pharmacist to be discussed in a case conference with the patient's GP within 4 weeks. DRPs with high priority were to be discussed first with the GP, followed by DRPs with medium priority, whereas DRPs with low priority were considerations with low urgency.

\section{Data classification}

Drugs were classified using the Anatomical Therapeutic Chemical (ATC) classification. Potential DRPs were classified using the D.O.C.U.M.E.N.T. classification system ${ }^{1,21,22}$ using the recently updated version. ${ }^{23,24}$

All coding and classification were independently undertaken by one investigator (H.K.) and a student investigator (Y.A.). When there were differences in coding, the investigators reached consensus in a case conference with a third investigator (either A.F. or M.B.).

\section{Outcome measures}

After 12 months $(t=12)$, medication records were collected to analyse the drug changes. The outcomes could only be assessed for patients with complete medication records available for at least 6 months. The total number of DRPs and recommendations were assessed by the main investigator (H.K.). Clinical relevance was assessed by the percentage of DRPs assigned a high priority, the percentage of recommendations for drug change and the percentage of implemented recommendations for drug change.

\section{Statistical analysis}

All data were analysed using databases (Microsoft Access 2007; Microsoft Corporation, Redmond, WA, USA) and statistical software (SPSS version 17.0; SPSS Inc., Chicago, IL, USA). Descriptive statistics were used for basic characteristics. Pearson chi-square tests were used for each categorical variable. An independent $t$-test was used for comparison of the mean number of DRPs per patient. A $P$-value $<0.05$ was considered statistically significant. Differences between the percentages of clinical relevant DRPs and recommendations identified during patient interviews and those identified by medication and clini- 
cal records were assessed by odds ratios (OR) and corresponding $95 \%$ confidence intervals.

\section{RESULTS AND DISCUSSION}

\section{Patient flow}

Patients were recruited for the intervention group in 10 community pharmacies. A total of 481 patients were eligible for participation in the intervention group of the study (Fig. 1). Of 396 patients were sent an invitation to participate. Of patients invited to participate, 188 patients $(47 \%)$ gave informed consent; 33 patients gave informed consent, but did not actually participate in the study. The reasons for not participating were loss of interest $(n=22)$, patient died $(n=4)$, health deterioration $(n=4)$ and hospital admission $(n=3)$. Finally, 155 patients were included in this analysis (response 39\%).

\section{Patient characteristics}

The median age of the patients was 76 years and $54 \%$ was women (Table 1). A mean of $4 \cdot 2$ diagnoses was registered per patient. The most common registered diagnoses were hypertension $(52 \%)$, diabetes mellitus (37\%) and hyperlipidaemia $(23 \%)$. The mean number of prescribed drugs per patient was $9 \cdot 0$ [range, 5-33]. The most commonly prescribed drug groups were agents acting on the renin-angiotensin system (ACE-inhibitors and AII antagonists) (72\%), antithrombotic agents (70\%) and lipid modifying agents (68\%).

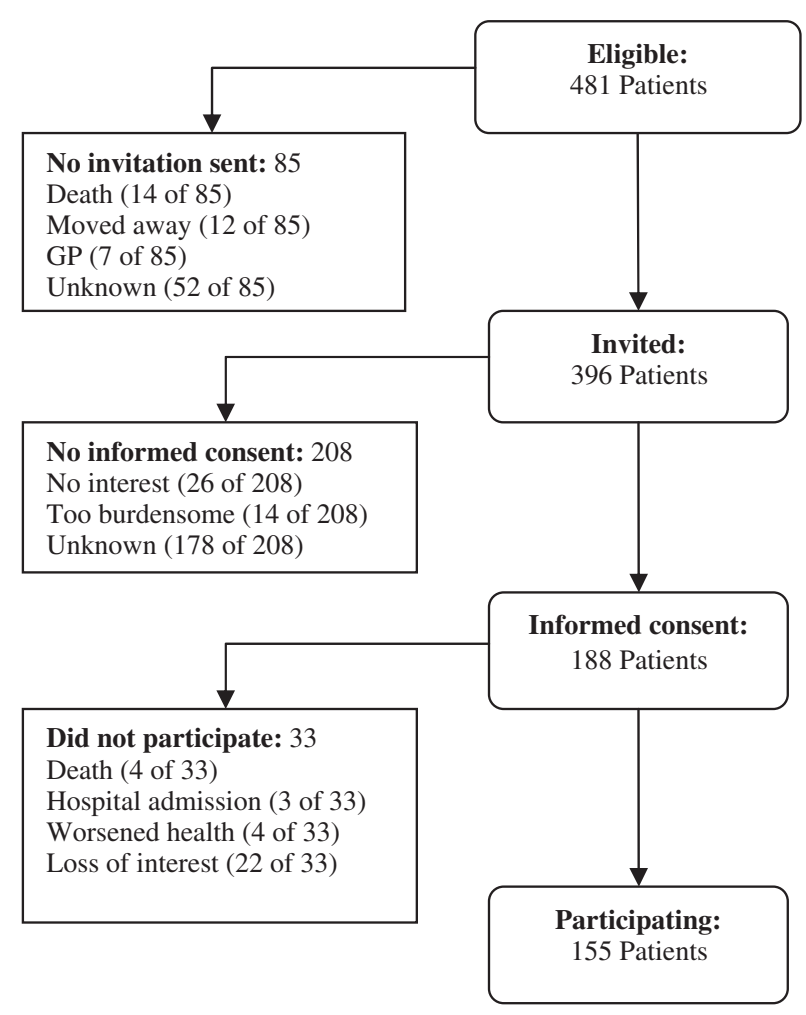

Fig. 1. Patient flow chart.

\section{DRPs and recommendations in general}

For 155 patients, a total of 1565 potential DRPs were identified, a mean of 10 DRPs per patient [range, 4-21] (Table 2). The most frequently observed types of DRPs were 'Drug Selection' (28\%), 'Undertreated '(26\%) and 'Monitoring required' (23\%). DRP subtypes were most often classified as 'No indication apparent' $(21 \%)$, 'Condition undertreated' (18\%) and 'Laboratory monitoring' (17\%).

Of 1565 recommendations, 905 (58\%) comprised a recommendation for drug change (mean, 5.8 per patient) (Table 3). The most common recommendations for drug change were 'Addition of a drug' (18\%) and 'Cessation of a drug' (13\%). Frequently recorded recommendations not involving a drug change were Laboratory Monitoring (19\%) and 'Adjustment of patient records' (13\%).

Of 905 recommendations for a drug change, 264 (29\%) recommendations were implemented as a drug change (Table 4). The most implemented drug changes were 'Cessation of a drug' (58, $23 \%)$ and 'Addition of a drug' (53, 21\%).

\section{DRPs and recommendations in patient interviews}

More than a quarter of DRPs and following recommendations (415; 27\%) were identified during patient interviews (Tables 2 and 3 ).

The DRP types 'Compliance' $(19 \%$ vs. $1.6 \%, P<0.01)$, 'Toxicity' (16\% vs. $2.5 \%, P<0.01)$, 'Over or underdose' $(14 \%$ vs. $9.7 \%, P=0.02)$ and 'Education' (1.7\% vs. $0.2 \%, P<0.01)$

Table 1. Baseline sociodemographic, medical and drug-related characteristics of 155 patients

Female $(n, \%)$

Age, year (median, interquartile range]

Number of prescription drugs (mean per

patient \pm SD)

Medical history, No. (\%)

Hypertension

Diabetes mellitus

Hyperlipidaemia

Coronary artery disease

Pulmonary disease

Arrhythmia

Cerebral vascular lesion, past or TIA

Cataract

Osteoporosis

Artrosis

Heart failure

Most prescribed drug groups (ATC), No. (\%)

Agents acting on the renin-angiotensin

system (C09)

Antithrombotic agents (B01A)

Lipid-modifying agents (C10A)

Beta blocking agents (C07A)

Drugs for peptic ulcer and GORD (A02B)

Calcium channel blockers (C08C)

Drugs for obstructive airway diseases (R03)

Drugs used in diabetes (A10)

Benzodiazepine derivatives (N05BA, N05CD)

High-ceiling diuretics $(\mathrm{C} 03 \mathrm{C})$

Low-ceiling diuretics (C03A, C03B, C03E)

\begin{tabular}{|c|c|}
\hline 84 & $54 \%$ \\
\hline 76 & $72-81$ \\
\hline $9 \cdot 0$ & 3.6 \\
\hline 75 & $52 \%$ \\
\hline 53 & $37 \%$ \\
\hline 33 & $23 \%$ \\
\hline 27 & $19 \%$ \\
\hline 23 & $16 \%$ \\
\hline 24 & $17 \%$ \\
\hline 25 & $17 \%$ \\
\hline 20 & $14 \%$ \\
\hline 16 & $11 \%$ \\
\hline 16 & $11 \%$ \\
\hline 12 & $8 \%$ \\
\hline 112 & $72 \%$ \\
\hline 109 & $70 \%$ \\
\hline 106 & $68 \%$ \\
\hline 80 & $52 \%$ \\
\hline 68 & $44 \%$ \\
\hline 50 & $32 \%$ \\
\hline 51 & $33 \%$ \\
\hline 48 & $31 \%$ \\
\hline 35 & $23 \%$ \\
\hline 33 & $21 \%$ \\
\hline 31 & $20 \%$ \\
\hline
\end{tabular}


Table 2. Comparison of number and type of DRPs identified from medication and clinical records compared to patient interviews for 155 patients

\begin{tabular}{|c|c|c|c|c|c|}
\hline \multirow[b]{2}{*}{ DRP type and subtype } & \multicolumn{2}{|c|}{$\begin{array}{l}\text { Medication } \\
\text { and clinical } \\
\text { records } \\
(N=1150 \\
\text { DRPs })\end{array}$} & \multicolumn{2}{|c|}{$\begin{array}{l}\text { Patient } \\
\text { interviews } \\
(N=415 \\
\text { DRPs })\end{array}$} & \multirow[b]{2}{*}{$P$-value } \\
\hline & $N$ & $\%$ & $N$ & $\%$ & \\
\hline D(rug selection) & 317 & 28 & 118 & 28 & $0 \cdot 74$ \\
\hline Duplication & 9 & $0 \cdot 8$ & 3 & $0 \cdot 7$ & $0 \cdot 91$ \\
\hline Drug interaction & 9 & $0 \cdot 8$ & 6 & $1 \cdot 5$ & $0 \cdot 23$ \\
\hline $\begin{array}{l}\text { Contra-indications } \\
\text { apparent }\end{array}$ & 67 & $5 \cdot 8$ & 7 & $1 \cdot 7$ & $<0 \cdot 01$ \\
\hline No indication apparent & 221 & 19 & 102 & 24 & $0 \cdot 02$ \\
\hline $\begin{array}{l}\text { Other drug selection } \\
\text { problem }\end{array}$ & 11 & $1 \cdot 0$ & 0 & $0 \cdot 0$ & $0 \cdot 05$ \\
\hline $\mathbf{O}$ (ver or underdose) & 111 & 10 & 58 & 14 & $0 \cdot 02$ \\
\hline $\begin{array}{l}\text { Prescribed dosage too } \\
\text { high }\end{array}$ & 24 & $2 \cdot 1$ & 9 & $2 \cdot 2$ & 0.92 \\
\hline $\begin{array}{l}\text { Prescribed dosage too } \\
\text { low }\end{array}$ & 60 & $5 \cdot 2$ & 12 & $2 \cdot 9$ & $0 \cdot 05$ \\
\hline $\begin{array}{l}\text { Incorrect or unclear } \\
\text { dosing instructions }\end{array}$ & 27 & $2 \cdot 3$ & 37 & 8.9 & $<0.01$ \\
\hline C(ompliance) & 18 & $1 \cdot 6$ & 78 & 19 & $<0.01$ \\
\hline Taking too little & 4 & $0 \cdot 3$ & 39 & $9 \cdot 4$ & $<0 \cdot 01$ \\
\hline Taking too much & 2 & $0 \cdot 2$ & 6 & $1 \cdot 4$ & $0 \cdot 02$ \\
\hline $\begin{array}{l}\text { Difficulty using dosage } \\
\text { form }\end{array}$ & 12 & $1 \cdot 0$ & 33 & $8 \cdot 0$ & $<0 \cdot 01$ \\
\hline $\mathbf{U}($ ndertreated) & 324 & 28 & 78 & 19 & $<0 \cdot 01$ \\
\hline Condition undertreated & 223 & 20 & 56 & 14 & $0 \cdot 04$ \\
\hline Condition untreated & 57 & $5 \cdot 0$ & 20 & $4 \cdot 8$ & 0.94 \\
\hline $\begin{array}{l}\text { Preventive therapy } \\
\text { required }\end{array}$ & 34 & $3 \cdot 0$ & 2 & 0.5 & $<0 \cdot 01$ \\
\hline $\mathbf{M}$ (onitoring) & 350 & 30 & 9 & $2 \cdot 2$ & $<0.01$ \\
\hline Laboratory monitoring & 260 & 23 & 1 & $0 \cdot 2$ & $<0 \cdot 01$ \\
\hline $\begin{array}{l}\text { Non-laboratory } \\
\text { monitoring }\end{array}$ & 90 & $7 \cdot 8$ & 8 & 1.9 & $<0 \cdot 01$ \\
\hline $\begin{array}{l}\text { E(ducation) or } \\
\text { information }\end{array}$ & 2 & $0 \cdot 2$ & 7 & $1 \cdot 7$ & $<0 \cdot 01$ \\
\hline $\begin{array}{l}\text { Disease management or } \\
\text { advice }\end{array}$ & 2 & $0 \cdot 2$ & 7 & $1 \cdot 7$ & $<0 \cdot 01$ \\
\hline $\mathbf{T}$ (oxicity) & 29 & $2 \cdot 5$ & 66 & 16 & $<0 \cdot 01$ \\
\hline $\begin{array}{l}\text { Toxicity, allergic } \\
\text { reaction or adverse } \\
\text { effect present }\end{array}$ & 29 & $2 \cdot 5$ & 66 & 16 & $<0 \cdot 01$ \\
\hline Mean per patient, SD & $7 \cdot 4 \pm 3 \cdot 0$ & & $\pm 2 \cdot 0$ & & $<0 \cdot 01$ \\
\hline
\end{tabular}

DRP, drug-related problem.

were relatively more identified during patient interviews, whereas 'Monitoring' (30.4\% vs. $2 \cdot 2 \%, P<0.01)$ and 'Undertreated' $(28 \%$ vs. $19 \%, P<0.01)$ were more frequently identified from medication and clinical records (Table 2). 'Toxicity, allergic reaction or adverse effect present' (16\% vs. 3\%, $P<0.01)$, 'Taking too little' $(9 \%$ vs. $0.3 \%, P<0.01)$ and 'Incorrect or unclear dosing instructions' (9\% vs. 2\%, $P<0.01)$ were the main DRP subtypes that were more frequently identified during patient interviews (Table 2). Examples of specific DRP subtypes in patient interviews are shown in Box 1.
Table 3. Comparisons of number and type of recommendations identified from medication and clinical records compared to patient interviews for 155 patients

\begin{tabular}{|c|c|c|c|c|c|}
\hline \multirow{2}{*}{$\begin{array}{l}\text { Type of } \\
\text { recommendation }\end{array}$} & \multicolumn{2}{|c|}{$\begin{array}{l}\text { Medication } \\
\text { and clinical } \\
\text { records } \\
(N=1150 \\
\text { recommen- } \\
\text { dations })\end{array}$} & \multicolumn{2}{|c|}{$\begin{array}{l}\text { Patient } \\
\text { interviews } \\
(N=415 \\
\text { recommen- } \\
\text { dations) }\end{array}$} & \multirow[b]{2}{*}{$P$-value } \\
\hline & $N$ & $\%$ & $N$ & $\%$ & \\
\hline $\begin{array}{l}\text { Recommendations for } \\
\text { drug change }\end{array}$ & 603 & 52 & 302 & 73 & $<0 \cdot 01$ \\
\hline Cessation of drug & 135 & 12 & 70 & 17 & $0 \cdot 01$ \\
\hline Dose increase & 75 & $6 \cdot 5$ & 23 & $5 \cdot 5$ & $0 \cdot 48$ \\
\hline Dose decrease & 38 & $3 \cdot 3$ & 25 & $6 \cdot 0$ & $0 \cdot 02$ \\
\hline Addition of drug & 219 & 19 & 56 & 14 & $0 \cdot 01$ \\
\hline Replacement of drug & 103 & $9 \cdot 0$ & 61 & 15 & $<0 \cdot 01$ \\
\hline $\begin{array}{l}\text { Dose frequency/ } \\
\text { schedule change }\end{array}$ & 28 & $2 \cdot 4$ & 46 & 11 & $<0 \cdot 01$ \\
\hline $\begin{array}{l}\text { Drug formulation } \\
\text { change }\end{array}$ & 5 & $0 \cdot 4$ & 19 & $4 \cdot 6$ & $<0 \cdot 01$ \\
\hline $\begin{array}{l}\text { Recommend dose } \\
\text { administration aid }\end{array}$ & 0 & $0 \cdot 0$ & 3 & $0 \cdot 7$ & $<0 \cdot 01$ \\
\hline Mean per patient, SD & $3.9 \pm 1.9$ & & $\pm 1 \cdot 6$ & & $<0.01$ \\
\hline Other recommendations & 547 & 48 & 113 & 27 & $<0 \cdot 01$ \\
\hline $\begin{array}{l}\text { Education/ } \\
\text { counselling session }\end{array}$ & 16 & $1 \cdot 4$ & 33 & $8 \cdot 0$ & $<0 \cdot 01$ \\
\hline $\begin{array}{l}\text { Monitoring: } \\
\text { Non-laboratory }\end{array}$ & 86 & $7 \cdot 5$ & 13 & $3 \cdot 1$ & $<0 \cdot 01$ \\
\hline $\begin{array}{l}\text { Monitoring: } \\
\text { Laboratory }\end{array}$ & 280 & 24 & 11 & $2 \cdot 7$ & $<0 \cdot 01$ \\
\hline $\begin{array}{l}\text { Adjustment of } \\
\text { patient records }\end{array}$ & 156 & 14 & 40 & $9 \cdot 6$ & $0 \cdot 04$ \\
\hline Other & 9 & $0 \cdot 8$ & 14 & $3 \cdot 4$ & $<0.01$ \\
\hline Mean per patient, SD & $3.5 \pm 1.9$ & & $\pm 0 \cdot 9$ & & $<0 \cdot 01$ \\
\hline
\end{tabular}

Table 4. Comparison of the clinical relevance of drug-related problems identified from medication and clinical records and during patient interviews

\begin{tabular}{|c|c|c|c|c|c|c|}
\hline \multirow{2}{*}{$\begin{array}{l}\text { Clinical relevance } \\
\text { of DRPs }\end{array}$} & \multicolumn{2}{|c|}{$\begin{array}{l}\text { Medication } \\
\text { and clinical } \\
\text { records }\end{array}$} & \multicolumn{2}{|c|}{$\begin{array}{l}\text { Patient } \\
\text { interviews }\end{array}$} & \multirow{2}{*}{$\begin{array}{l}\text { OR } \\
{[C I \text { 95\%] }}\end{array}$} & \multirow[b]{2}{*}{$P$-value } \\
\hline & $N$ & $\%$ & $N$ & $\%$ & & \\
\hline Overall (reference) & 1150 & & 415 & & ref & \\
\hline With high priority & 445 & 39 & 219 & 53 & $1 \cdot 8[1 \cdot 4-2 \cdot 2]$ & $<0 \cdot 01$ \\
\hline $\begin{array}{l}\text { With } \\
\text { recommendations } \\
\text { for drug change }\end{array}$ & 603 & 52 & 302 & 73 & $2 \cdot 4[1 \cdot 9-3 \cdot 1]$ & $<0 \cdot 01$ \\
\hline $\begin{array}{l}\text { With implemented } \\
\text { recommendations } \\
\text { for drug change }\end{array}$ & 145 & 13 & 120 & 29 & $2 \cdot 8[2 \cdot 1-3 \cdot 7]$ & $<0 \cdot 01$ \\
\hline
\end{tabular}

DRP, drug-related problem. 
BOX 1. EXAMPLES OF DRPS IDENTIFIED DURING PATIENT INTERVIEWS

\begin{tabular}{|c|c|c|c|}
\hline $\begin{array}{l}\text { Gender, } \\
\text { age (years) }\end{array}$ & Example of DRP & DRP subtype & $\begin{array}{l}\text { Assigned } \\
\text { priority }\end{array}$ \\
\hline 3, 75 & $\begin{array}{l}\text { Despite use of two antihypertensives (nifedipine } 30 \mathrm{mg} \text { retard once daily, } \\
\text { candesartan } 16 \mathrm{mg} \text { once daily), measurement at home shows a very high } \\
\text { systolic blood pressure of } 198 \mathrm{mmHg}\end{array}$ & Condition undertreated & High \\
\hline ㅇ, 79 & $\begin{array}{l}\text { Uses nitroglycerine almost daily at } 5 \text { PM because of dyspnoe. She experiences } \\
\text { flushes. Also, uses isosorbide mononitrate } 60 \mathrm{mg} \text { once daily and diltiazem } \\
60 \mathrm{mg} \text { three times a day. Next to cardiovascular medication uses a combined } \\
\text { budesonide and salmeterol inhaler two times a day }\end{array}$ & Condition undertreated & High \\
\hline 3,83 & $\begin{array}{l}\text { Uses betamethason ointment every day without using an emollient. Complains } \\
\text { about delayed healing of wounds }\end{array}$ & Condition undertreated & High \\
\hline ㅇ, 78 & $\begin{array}{l}\text { Has restarted alendronic acid without consulting physician. Thought this was a } \\
\text { preventive measure during use of iron tablets. Stopped taking prednisolone a } \\
\text { half year before the interview and does not suffer from osteoporosis according } \\
\text { to the clinical data }\end{array}$ & No indication apparent & High \\
\hline 우 70 & $\begin{array}{l}\text { Takes levothyroxin at } 9 \text { AM Did not know that this has to be taken half an hour } \\
\text { before breakfast }\end{array}$ & $\begin{array}{l}\text { Incorrect or unclear dosing } \\
\text { instructions }\end{array}$ & High \\
\hline q, 65 & Intermittently uses furosemide $60 \mathrm{mg}$ for facial oedema & No indication apparent & Medium \\
\hline q, 75 & $\begin{array}{l}\text { Uses a coumarin and experiences severe bleeding during blood sampling. Suffers } \\
\text { from itching in the evening, especially when the heater goes on and suspects } \\
\text { this is an adverse effect }\end{array}$ & $\begin{array}{l}\text { Toxicity, allergic reaction or } \\
\text { adverse effect present }\end{array}$ & Medium \\
\hline 0,76 & $\begin{array}{l}\text { Experiences hoarseness during use of beclomethason } 100 \mu \mathrm{g} \text { three doses at once } \\
\text { after breakfast }\end{array}$ & $\begin{array}{l}\text { Toxicity, allergic reaction or } \\
\text { adverse effect present }\end{array}$ & Medium \\
\hline 3,73 & $\begin{array}{l}\text { Uses metformine } 850 \mathrm{mg} \text { once daily instead of the prescribed } 3 \text { times a day } \\
\text { because of gastrointestinal problems. Is of the opinion that he gets prescribed } \\
\text { too many medicines. Except metformin uses a long-acting insulin before the } \\
\text { night and a short acting insulin before meals }\end{array}$ & Taking too little & Medium \\
\hline 3,76 & $\begin{array}{l}\text { Uses six different medicines (fosinopril pantoprazol, metoprolol, acetylsalicylic } \\
\text { acid, atorvastatin and alfuzosin) at different dosing moments, whereas these } \\
\text { could be taken at the same time }\end{array}$ & $\begin{array}{l}\text { Incorrect or unclear dosing } \\
\text { instructions }\end{array}$ & Low \\
\hline + 75 & $\begin{array}{l}\text { Uses half a tablet of } 80 \mathrm{mg} \text { sotalol twice daily, whereas } 40 \mathrm{mg} \text { tablets are } \\
\text { available and would be more convenient }\end{array}$ & $\begin{array}{l}\text { Incorrect or unclear dosing } \\
\text { instructions }\end{array}$ & Low \\
\hline + 79 & Experiences coughing by captopril. However, this is not disturbing & $\begin{array}{l}\text { Toxicity, allergic reaction or } \\
\text { adverse effect present }\end{array}$ & Low \\
\hline
\end{tabular}

DRP, drug-related problem.

'Dose frequency/schedule change' $(11 \%$ vs. $2.4 \%, P<0.01)$ and 'Drug formulation change' ( $5 \%$ vs. $0.4 \%, P<0.01$ ) were more often recommended based on patient interviews (Table 3). Both recommendations were also more often implemented: 'Dose frequency/schedule change' $(7 \cdot 4 \%$ vs. $1.5 \%, P<0.01)$ and 'Drug formulation change' $(1.4 \%$ vs. $0.1 \%, P=0.03)$.

\section{Clinical relevance of DRPs}

Drug-related problems assigned a high priority were more likely to be identified during patient interviews than from medication and clinical records $(\mathrm{OR}=1 \cdot 8[1 \cdot 4-2 \cdot 2] ; P<0 \cdot 01)$ (Table 4). Examples of DRP subtypes identified during patient interviews with high, medium and low priority are shown in Box 1.

Furthermore, DRPs followed by recommendations for a drug change were more likely to be identified during patient interviews $(\mathrm{OR}=2 \cdot 4$ [1.9-3.1], $P<0 \cdot 01)$ (Table 3 and 4$)$.

Finally, DRPs followed by implemented recommendations for drug change were more likely to be identified during patient interviews ( $28 \%$ vs. $12 \%$; OR $=2 \cdot 8$ [2.1-3.7]; $P<0 \cdot 01)$ (Table 4$)$.
This study shows that patient interviews at home contribute significantly to the identification of clinical relevant DRPs. Not only were more than a quarter of all DRPs identified during patient interviews, the DRPs identified during patient interviews were also assigned higher priorities and more frequently led to recommendations involving a drug change and were more enacted compared to DRPs identified from clinical and medication records.

The relative contribution of patient interviews to identification of all DRPs in our study was comparable to findings of Krska (29\%) in primary care. ${ }^{6}$ Studies with patient interviews in other settings reported higher percentages (GP's office $73 \%$, hospital 40\%), but the intervention and population in these studies were also quite different. ${ }^{3,7}$ In addition, in our study, $53 \%$ of the DRPs in patient interviews were assigned a high priority, and $73 \%$ were followed by a recommendation involving a drug change. The only study that previously looked into the clinical relevance of DRPs identified by patient interviews was performed in hospitalized patients and assessed that $65 \%$ of these DRPs were of high relevance. $^{3}$ 
'Toxicity, allergic reaction or adverse effect present' was the most frequent DRP subtype that was identified in patient interviews. This was also the most common pharmaceutical care issue in the study of Krska. ${ }^{6}$ This finding gives support to the assumption that the concerns of the patient were sufficiently addressed by the community pharmacists in the home visits. This is not always obvious, because analysis of taped consultations from the HOMER study suggested that pharmacist reviewers were primarily concerned with compliance and knowledge of drugs. ${ }^{16,25}$

'Taking too little' was the second DRP subtype that was significantly more identified during patient interviews. Compliance issues are mentioned as DRPs in many studies with patient interviews. ${ }^{6,26,27}$ Sturgess showed that clinical medication review including patient interviews even improved compliance measured by self-reporting and refill rate. ${ }^{27}$ Repeated domiciliary visits after initial medication review may also enhance compliance as was measured by pill counts. ${ }^{28}$ Their explanation was that compliance issues might be discussed easier in a patient's home than in a busy community pharmacy. ${ }^{26}$

'Incorrect or unclear dosing instructions' was the third DRP subtype that was identified significantly more in patient interviews. The corresponding recommendation 'Dose frequency/ schedule change' was often implemented. For many patients, minor changes in their dose schedule can diminish the frequency of dosing. As shown in an example in Box 1, some patients are taking medication throughout the whole day, whereas these could be taken at the same time. In an earlier domiciliary interviewing study, half of the patients judged that medication management was a major daily preoccupation, and spouses were often required to assist. ${ }^{28}$

It could be questioned whether patient interviews at home reveal additional or other type of DRPs than patient interviews in a consulting area in the community pharmacy or GP practice; however, this was not the aim of this study. On the one hand, patient interviews at home may elicit more and other DRPs, because patients might feel more comfortable at home and therefore are more likely to share their experiences and concerns about their medicines. This is illustrated by the finding that compliance issues and adverse effects were frequently identified by patient interviews in our study and Krska's study. ${ }^{6}$ Furthermore, all medicines were available at home, whereas patients invited to the pharmacy or GP practice might forget to bring part of their medicines, especially those that are used intermittently. ${ }^{8}$ Finally, certain medication-risk factors, for example, lack of medication administration routine, multiple storage locations, hoarding and medication storage conditions seem only to be identified by home visits. ${ }^{26,28-30}$ On the other hand, costs of home visits are also higher. Future studies should focus on cost effectiveness of patient interviews at home compared to interviews conducted at a GP practice or pharmacy.

This study had several strengths. Firstly, the intervention in our study comprised a clinical medication review meaning that all data were available to conduct a medication review. Next to the availability of all clinical records of the patients, additional laboratory values and blood pressure values were obtained as part of the RCT. Despite the availability of all these data, more than a quarter of all DRPs were identified from patient interviews. Secondly, a very detailed and accurate description of DRPs was possible, because all pharmaceutical care plans and reports of patient interviews were electronically sent by the community pharmacists to the pharmacist reviewers. This made it also possible to distinguish very clearly between DRPs identified from medication and clinical records and from patient interviews without any overlapping. Thirdly, DRPs were assigned a priority by the pharmacist reviewers' panel, and this could be seen as an indicator of clinical relevance. Fourthly, patient interviews were conducted by community pharmacists. More than half of the DRPs identified from these patient interviews had a high priority. In an earlier study, in which patient interviews were conducted by pharmacy or practice assistants, only a quarter of identified DRPs had potential clinical relevance. ${ }^{31}$ Finally, the use of experienced pharmacist reviewers probably led to more complete and standardized pharmaceutical care plans.

There are some limitations associated with this study. Firstly, a considerable part of the invited patients $(53 \%)$ did not give informed consent. However, our target group is an older population who have a considerable disease burden. In particular, the extra laboratory monitoring that was part of the study was not appreciated by many potential participants. Secondly, community pharmacists had a short course (2-days) in medication review and had limited experience in conducting medication reviews. Finally, each initial pharmaceutical care plan by a community pharmacist was adjusted and completed by well-trained pharmacist reviewers who had no relationship with patients. Although standardization is a strength of the study, it may limit generalizability to daily clinical practice where pharmacists might identify less DRPs, but have a relationship with patients. Future research should further elaborate on the role of both the community pharmacists and the pharmacist reviewers regarding the identification of DRPs.

\section{WHAT IS NEW AND CONCLUSION}

Patient interviews by community pharmacists have a major contribution in the identification of DRPs. This implies that, in general, medication review without a patient interview may lead to fewer clinical relevant recommendations.

\section{ACKNOWLEDGEMENTS}

This study was conducted in pharmacies of LLOYDS Apotheken. The study received unrestricted research funding by the Royal Dutch Association of Pharmacists (KNMP), Astra-Zeneca and the healthcare insurance companies Achmea and Menzis. This work was performed totally independently from these funders. The authors like to thank all ten participating community pharmacies, L.A. Schul of LLOYDS Apotheken for her coordinating activities, J.M. Krijger-Dijkema as a pharmacist reviewer and Y. Amarouchi, student, for his contribution to the data classification.

\section{CONFLICT OF INTEREST}

All authors declare that they have no conflict of interests that are directly relevant to the content of this study. 


\section{REFERENCES}

1. Kwint HF, Faber A, Gussekloo J, Bouvy ML. Effects of medication review on drugrelated problems in patients using automated drug-dispensing systems: a pragmatic randomized controlled study. Drugs Aging, 2011;28:305-314.

2. Denneboom W, Dautzenberg MG, Grol R, De Smet PA. Treatment reviews of older people on polypharmacy in primary care: cluster controlled trial comparing two approaches. Br J Gen Pract, 2007;57:723-731.

3. Viktil KK, Blix HS, Moger TA, Reikvam A. Interview of patients by pharmacists contributes significantly to the identification of drug-related problems (DRPs). Pharmacoepidemiol Drug Saf, 2006;15:667-674.

4. Zermansky AG, Petty DR, Raynor DK, Freemantle N, Vail A, Lowe CJ. Randomised controlled trial of clinical medication review by a pharmacist of elderly patients receiving repeat prescriptions in general practice. BMJ 2001;323:1340-1343.

5. Sorensen L, Stokes JA, Purdie DM, Woodward M, Elliott R, Roberts MS. Medication reviews in the community: results of a randomized, controlled effectiveness trial. Br J Clin Pharmacol, 2004; 58:648-664.

6. Krska J, Cromarty JA, Arris F et al. Pharmacist-led medication review in patients over 65: a randomized, controlled trial in primary care. Age Ageing, 2001;30:205-211.

7. Jameson JP, VanNoord GR. Pharmacotherapy consultation on polypharmacy patients in ambulatory care. Ann Pharmacother, 2001;35:835-840.

8. Nathan A, Goodyer L, Lovejoy A, Rashid A. 'Brown bag' medication reviews as a means of optimizing patients' use of medication and of identifying potential clinical problems. Fam Pract, 1999;16:278-282.

9. Clyne W, Blenkinsopp A, Seal R. A guide to medication review 2008. Available at: http://www.npci.org.uk/medicines_management/review/medireview/library/ library_good_practice_guide1.php (accessed 19 April 2010).

10. Castelino RL, Bajorek BV, Chen TF. Retrospective evaluation of home medicines review by pharmacists in older Australian patients using the medication appropriateness index. Ann Pharmacother, 2010;44: 1922-1929.
11. Castelino RL, Bajorek BV, Chen TF. Are interventions recommended by pharmacists during Home Medicines Review evidence-based? J Eval Clin Pract, 2011;17:104-110.

12. Castelino RL, Hilmer SN, Bajorek BV, Nishtala P, Chen TF. Drug Burden Index and potentially inappropriate medications in community-dwelling older people: the impact of home medicines review. Drugs Aging, 2010;27:135-148.

13. Latif A, Pollock K, Boardman HF. The contribution of the medicines use review (MUR) consultation to counseling practice in community pharmacies. Patient Educ Couns, 2011;83:336-344.

14. Grymonpre RE, Williamson DA, Montgomery PR. Impact of a pharmaceutical care model for non-institutionalised elderly: results of a randomised, controlled trial. Int J Pharm Pract, 2001;9:235241.

15. Bernsten C, Bjorkman I, Caramona M et al. Improving the well-being of elderly patients via community pharmacy-based provision of pharmaceutical care: a multicentre study in seven European countries. Drugs Aging, 2001;18:63-77.

16. Holland R, Lenaghan E, Harvey I et al. Does home based medication review keep older people out of hospital? The HOMER randomised controlled trial $B M J$ 2005;330:293-295.

17. Buurma H, Bouvy ML, De Smet PA, Floor-Schreudering A, Leufkens HG, Egberts AC. Prevalence and determinants of pharmacy shopping behaviour. J Clin Pharm Ther, 2008;33:17-23.

18. Krska J, Avery AJ. Evaluation of medication reviews conducted by community pharmacists: a quantitative analysis of documented issues and recommendations. Br J Clin Pharmacol, 2008;65:386-396.

19. Cipolle RJ. Pharmaceutical care practice: the clinician's guide. New York, London: McGraw-Hill, Medical Pub. Division, 2004.

20. Leendertse AJ, de Koning FH, Goudswaard AN et al. Preventing hospital admissions by reviewing medication (PHARM) in primary care: design of the cluster randomised, controlled, multi-centre PHARM-study. BMC Health Serv Res, 2011;11:4
21. Tenni P, Stafford A, Peterson G, Jackson S. A comparison of drug-related problems identified in RMMRs and HMRs. Aust Pharm 2007;26:414-420.

22. Rasmussen M, Stafford AC, Tenni PC et al. Drug-related problems identified in medication reviews by Australian pharmacists. Pharm World Sci, 2009;31:216-223.

23. Williams M, Peterson GM, Tenni PC et al. Drug-related problems detected in Australian Community Pharmacies: the PROMISe Trial. Ann Pharmacother, 2011;45: 1067-1076.

24. Williams M, Peterson GM, Tenni PC, Bindoff IK, Stafford AC. DOCUMENT: a system for classifying drug-related problems in community pharmacy. Int J Clin Pharm, 2012;34:43-52.

25. Salter C, Holland R, Harvey I, Henwood K. "I haven't even phoned my doctor yet." The advice giving role of the pharmacist during consultations for medication review with patients aged 80 or more: qualitative discourse analysis. BMJ, 2007;334:1101.

26. Begley S, Livingstone $\mathrm{C}$, Hodges $\mathrm{N}$, Willamson V. Impact of domiciliary pharmacy visits on medication management in an elderly population. Int J Pharm Pract 1997;5:111-121.

27. Sturgess IK, McElnay JC, Hughes CM, Crealey G. Community pharmacy based provision of pharmaceutical care to older patients. Pharm World Sci, 2003;25:218-226.

28. Coleman DJ, Portlock J, Brown D. Delivering domiciliary pharmaceutical care from a health centre pharmacy. Int I Pharm Pract, 2001;9:127-137.

29. Sorensen L, Stokes JA, Purdie DM, Woodward M, Roberts MS. Medication management at home: medication-related risk factors associated with poor health outcomes. Age Ageing, 2005;34:626-632.

30. Sorensen L, Stokes JA, Purdie DM, Woodward M, Roberts MS. Medication management at home: medication risk factor prevalence and inter-relationships. J Clin Pharm Ther, 2006;31:485-491.

31. Denneboom W, Dautzenberg MG, Grol R, De Smet PA. User-related pharmaceutical care problems and factors affecting them: the importance of clinical relevance. J Clin Pharm Ther, 2005;30:215-223. 